\title{
Dysfunctional Beliefs about Sleep in Cancer Patients Can Mediate the Effect of Fear of Progression on Insomnia
}

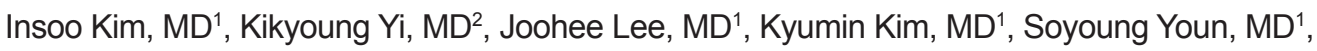 \\ Sooyeon Suh, $\mathrm{PhD}^{3}$, Jiyeon Kim, $\mathrm{PhD}^{4}$, Jung Mun Choi, BA ${ }^{1}$, Seockhoon Chung, MD, PhD ${ }^{1}$ \\ 'Department of Psychiatry, Asan Medical Center, University of Ulsan College of Medicine, Seoul, Korea \\ 2Department of Psychiatry, Yongin Mental Health, Yongin, Korea \\ ${ }^{3}$ Department of Psychology, Sungshin Women's University, Seoul, Korea \\ ${ }^{4}$ Department of Art Therapy, Hanyang Cyber University, Seoul, Korea
}

Received: October 21, 2019

Revised: November 1, 2019

Accepted: November 5, 2019

Correspondence

Seockhoon Chung, MD, PhD

Department of Psychiatry,

Asan Medical Center,

University of Ulsan College of Medicine,

86 Olympic-ro 43-gil, Songpa-gu,

Seoul 05505, Korea

Tel +82-2-3010-3411

Fax +82-2-485-8381

E-mail_schung@amc.seoul.kr

ORCID

Insoo Kim

https://orcid.org/0000-0002-0238-5042

Kikyoung Yi

https://orcid.org/0000-0003-1410-7901

Joohee Lee

https://orcid.org/0000-0002-3114-1944

Kyumin Kim

https://orcid.org/0000-0003-1203-1157

Soyoung Youn

https://orcid.org/0000-0003-4983-4017

Sooyeon Suh

https://orcid.org/0000-0003-0644-8634

Jiyeon Kim

https://orcid.org/0000-0001-7406-3176

Jung Mun Choi

https://orcid.org/0000-0002-2599-7092

Seockhoon Chung

https://orcid.org/0000-0002-9798-3642
Background and Objective The role of the dysfunctional belief about sleep is important for the development of insomnia among cancer patients. This study intended to investigate whether dysfunctional belief about sleep mediates the relationship between fear of progression and insomnia in cancer patients.

Methods Three hundred and thirty-seven cancer patients participated in our study. Dysfunctional belief about sleep, severity of insomnia, depression, fear of progression, and anxiety were measured using the following questionnaires: Cancer-related Dysfunctional Beliefs about Sleep (C-DBS); Insomnia Severity Index (ISI); Patient Health Questionnaire-9 (PHQ-9); Fear of Progression (FoP); and the state subcategory of State and Trait Anxiety Inventory (STAI). Path analysis was used to clarify the relationships among the variables. Since C-DBS consists of two items, i.e., Q1-immune \& Q2-recurrence, we implemented an additional path analysis including these variables separately.

Results C-DBS mediated the effect of FoP $(\beta=0.36, p<0.001)$ and patient sex $(\beta=0.13, p=$ $0.009)$ on ISI. PHQ-9 $(\beta=0.32, p<0.001)$ and STAI $(\beta=-0.09, p=0.071)$ had a direct influence on ISI scores. In our second path analysis, Q1-immune item mediated the effect of FoP $(\beta=0.19, \mathrm{p}<$ $0.001)$ on ISI, and Q2-recurrence item mediated the effect of FoP $(\beta=0.23, \mathrm{p}<0.001)$ and patient $\operatorname{sex}(\beta=0.09, \mathrm{p}=0.019)$ on ISI.

Conclusions Our path analysis model indicated that C-DBS mediates the effect of FoP and patient sex on ISI. Our second path analysis results suggested that there could be an internal process of Q1 and Q2 item. Efforts to reduce dysfunctional beliefs should be considered as well as management of fear of progression for better sleep of cancer patients.

Sleep Med Res 2019;10(2):83-89

Key Words Sleep-related cognition, Insomnia, Cancer, Fear.

\section{INTRODUCTION}

Cancer patients frequently experience insomnia as well as other psychiatric symptoms including depression and other emotional discomfort [1]. In particular, insomnia is a common symptom in cancer patients with a prevalence of being approximately three times greater compared to that in healthy adults [2]. Insomnia itself may be nerve wrecking, but moreover it can actually cause health problems. A previous study suggested that older adults who showed prolonged sleep latencies, poor sleep efficiency or abnormal percentage of REM sleep have an increased risk of mortality [3].

Insomnia in cancer patients differs from insomnia in the general, healthy population. Sleeprelated cognition distortion in cancer patients has been thought to be characteristic compared to that in the general population, and this concept led to the development of a tool, i.e., Cancer- 
related Dysfunctional Beliefs about Sleep (C-DBS) scale, that assesses cognition distortion, i.e., "cancer-related dysfunctional beliefs" about sleep in that specific population [4,5]. This scale includes items that involve patient's concern about the consequences of insomnia, such as immune decline (Q1-immune item) or cancer recurrence/metastasis (Q2-recurrence item). We have recently endeavored to clarify the role of cancer-related dysfunctional beliefs about sleep in cancer patients and one of our previous studies suggests that these beliefs have a significant association with the severity of insomnia, independent of depression [6]. In an earlier study, these beliefs exhibited a significant correlation with the severity of insomnia and the degree of fear of disease progression in cancer patients [4].

Fear of progression is defined as "patients' fear that illness will progress with all its biopsychosocial consequences, or that it will recur," and although it is a rational reaction to the real threat of cancer with which patients are confronted, elevated levels of fear of progression can be dysfunctional [7]. Higher levels of fear of progression were related with a higher risk for poor sleep quality in cancer survivors [8]. The concept of fear of progression along with cancer-related dysfunctional beliefs about sleep is important in terms of understanding the distress that cancer patients go through from a cognitive perspective. Treatment of cancer patients is now considered a multidimensional process and recognizing distress is certainly a vital part of achieving that goal. Fear of progression and cancer-related dysfunctional beliefs about sleep in cancer patients showed a significant correlation in our previous study [4]. In the same study, women's C-DBS scores appeared to be higher than men's and a negative correlation between patient age and Q2-recurrence item score was found. Also, Q1-immune item and Q2-recurrence item scores were highly correlated with each other, i.e., Q1-immune item being significantly correlated with insomnia severity and fear of progression, and Q2-recurrence item with insomnia, depression, and fear of progression.

Therefore, we hypothesized that cancer-related dysfunctional beliefs about sleep might mediate the effect of depression, insomnia, anxiety or fear of progression on insomnia severity. We also postulated that those variables may have direct effects on insomnia severity along with patient age or sex.

\section{METHODS}

\section{Participants}

Three hundred and thirty-seven patients diagnosed with various types of cancer and who visited the Sleep Clinic for Cancer Patients of Asan Medical Center between January 2017 and February 2018 participated in our study. The medical records of these patients were retrospectively reviewed. The study protocol was approved by the Institutional Review Board of Asan Medical Center (2018-0618), and a previous study had been pub- lished using the same data [5].

Demographic information included patient age, sex, type of cancer, cancer stage, type of treatment, surgical history, and psychiatric diagnosis. All of the participants completed the C-DBS, Insomnia Severity Index (ISI), Patient Health Questionnaire-9 (PHQ-9), Fear of Progression (FoP), and the State-Trait Anxiety Inventory Score (STAI). The rating scale scores are shown in Table 1. Participants were over 18 years of age, capable of independently completing the rating scales, had no difficulties in communication, and no evidence of delirium or major psychotic disorders. The psychiatric diagnosis was made at the time of the first visit to the Sleep Clinic for Cancer Patients.

\section{Measures}

\section{C-DBS}

C-DBS is a tool developed to assess dysfunctional beliefs in cancer patients with sleep disturbance [4]. It consists of two questions which address dysfunctional beliefs that cancer patients may have, i.e., "My immune system will have serious problems if I don't go to sleep at a certain time" (Q1-immune) and "If I don't sleep well at night, my cancer may recur or metastasize" (Q2-recurrence). Patients were asked to rate each question on a $0-10$ scale. The sum of Q1 and Q2 scores refers to the total C-DBS score.

\section{ISI}

ISI is a self-report measure consisting of seven items assessing insomnia symptoms. The severity of insomnia problems, satisfaction with the current sleep pattern, interference with one's daily functioning, noticeability of impairment, and the degree of distress or concern are assessed. Each item is rated on a 0-4 Likert scale, with higher scores implying more severe symptoms [9]. The Korean version of the ISI, which was used in this study, showed good reliability and validity in a previous study [10].

\section{PHQ-9}

PHQ-9 is a brief self-report measure consisting of nine items assessing the severity of depression. Depressive symptoms such as anhedonia, depressed mood, insomnia, tiredness, loss of appetite, feeling guilt, inability to concentrate, psychomotor retardation, and suicidal/self mutilating idea are assessed. Each item is rated on a $0-3$ scale, with higher scores implying more severe depression [11].

\section{FoP}

FoP is a self-reporting psychological questionnaire measuring the fear of progression in chronically ill patients. It contains 43 items that cover five dimensions, i.e., affective reactions, partnership/family issues, occupation, loss of autonomy, and coping with anxiety. Each item is rated on a 1-5 scale, and providing two total scores, one for all anxiety subscales and another for the coping scale [12]. In our study, the Korean version of the 
Table 1. Demographic characteristics of subjects

\begin{tabular}{|c|c|}
\hline Variable & Value \\
\hline \multicolumn{2}{|l|}{ Subjects $(\mathrm{n}=337)$} \\
\hline Male & $108(32.0)$ \\
\hline Age (yr) & $54.0 \pm 11.8$ \\
\hline \multicolumn{2}{|l|}{ Cancer type } \\
\hline Breast & $149(44.2)$ \\
\hline Lung & $37(11.0)$ \\
\hline Pancreatic and biliary tract & $34(10.0)$ \\
\hline Gastro-esophageal & $28(8.3)$ \\
\hline Colorectal & $25(7.4)$ \\
\hline Hematologic & $16(4.7)$ \\
\hline Gynecologic & $11(3.3)$ \\
\hline Urinary tract & $10(3.0)$ \\
\hline Liver & $9(2.7)$ \\
\hline Kidney & $7(2.1)$ \\
\hline Thyroid & $4(1.2)$ \\
\hline Others & $7(2.1)$ \\
\hline \multicolumn{2}{|l|}{ Cancer stages $(\mathrm{n}=316)^{*}$} \\
\hline Stage 0 & $7(2.3)$ \\
\hline Stage I & $62(20.0)$ \\
\hline Stage II & $85(27.4)$ \\
\hline Stage III & $54(17.4)$ \\
\hline Stage IV & $68(21.9)$ \\
\hline Not yet confirmed & $34(11.0)$ \\
\hline \multicolumn{2}{|l|}{ History of treatment } \\
\hline Chemotherapy & $186(55.2)$ \\
\hline Radiotherapy & $78(23.1)$ \\
\hline Hormone therapy & $61(18.1)$ \\
\hline Immunotherapy & $12(3.6)$ \\
\hline Surgical operation & $261(77.4)$ \\
\hline \multicolumn{2}{|l|}{ Psychiatric diagnosis } \\
\hline No diagnosis & $29(8.6)$ \\
\hline Acute stress reaction/adjustment disorder & $24(7.1)$ \\
\hline Major depressive disorder & $118(35.0)$ \\
\hline Insomnia & $115(34.1)$ \\
\hline $\begin{array}{l}\text { Anxiety disorder/somatic symptom } \\
\text { disorder }\end{array}$ & $32(9.5)$ \\
\hline Mixed anxiety and depressive disorder & $15(4.5)$ \\
\hline Others & $4(1.2)$ \\
\hline \multicolumn{2}{|l|}{ Rating scale scores } \\
\hline Insomnia Severity Index & $16.0 \pm 6.1$ \\
\hline $\begin{array}{l}\text { Cancer-related Dysfunctional Beliefs } \\
\text { about Sleep }\end{array}$ & $11.9 \pm 5.7$ \\
\hline Q1-immune & $6.1 \pm 3.0$ \\
\hline Q2-recurrence & $5.9 \pm 3.2$ \\
\hline Patient Health Questionnaire-9 & $11.8 \pm 6.5$ \\
\hline Fear of Progression & $36.2 \pm 11.7$ \\
\hline $\begin{array}{l}\text { State subcategory of State-Trait Anxiety } \\
\text { Inventory }\end{array}$ & $38.6 \pm 11.4$ \\
\hline
\end{tabular}

Data are expressed as mean \pm standard deviation or $n(\%)$.

*Among patients with cancer graded using the TNM staging system. short form of FoP was used [13].

\section{STAI}

The State-Trait Anxiety Inventory (STAI) is a self-report psychological inventory which includes questions about the state and trait anxiety. It is based on a 4-point Likert scale and higher scores are correlated with higher levels of anxiety [14]. In our study, only the state anxiety subcategory (STAI-S) of the full scale was utilized.

\section{Statistical Analysis}

All of the data, including the mean, standard deviation of continuous variables, frequency, and percentage of categorical variables were summarized. In order to evaluate the association among the measures, Pearson's correlation coefficients were calculated.

Path analysis and structural equation modeling were used to evaluate the relationship of patient age, sex, dysfunctional beliefs about sleep, severity of insomnia, depression, anxiety, and fear of progression. Assuming that C-DBS works as a possible mediator among PHQ-9, FoP, STAI-S, age, sex, ISI, and C-DBS, we constructed hypothetical-based models and evaluated how real data fit them based on the path analysis and structural equation models. The direct effects of STAI-S, age, and sex on ISI were estimated. Also, the indirect effects of PHQ-9, FoP, age and sex on ISI through C-DBS were computed. In our second path analysis, the indirect effects of FoP on ISI through Q1-immune item and Q2-recurrence item. Global model fit was assessed using various measures such as the chi-square goodness of fit, the root mean square error of approximation (RMSEA), the root mean square residual (RMR), Normed Fit Index (NFI), etc. Missing values in the dataset were managed using the multiple Bayesian imputation method.

The Statistical Package for Social Sciences (SPSS) version 21 (IBM Corp., Armonk, NY, USA) was used for correlation and regression analyses with the level of significance being defined as $\mathrm{p}<0.05$ in the two-tailed tests. AMOS22 was used for the path analysis.

\section{RESULTS}

\section{Demographics}

Demographic information about the patients is shown in Table 1 . Among the participants, $32 \%$ were male and the overall average age was 54 years. The most common cancer type was breast cancer (44.2\%), followed by lung cancer (11.0\%), pancreatic and biliary tract cancer (10.0\%), and gastro-esophageal cancer (8.3\%). Regarding cancer stages, the percentages of stage I to IV were approximately even except for stage $0(2.3 \%)$. More than half of the patients received chemotherapy (55.2\%), some radiotherapy (23.1\%), and hormone therapy (18.1\%). The majority has undergone surgery (77.4\%). The most common psychi- 
atric diagnoses were major depressive disorder (35.0\%) and insomnia (34.1\%), followed by anxiety/somatic symptom disorder (9.5\%) and acute stress reaction/adjustment disorder (7.1\%). The mean ISI score was $16.0 \pm 6.1$, and the C-DBS, Q1-immune, Q2-recurrence, PHQ-9, FoP, and STAI-S scores were 11.9 \pm 5.7, $6.1 \pm 3.0,5.9 \pm 3.2,11.8 \pm 6.5,36.2 \pm 11.7$, and $38.6 \pm 11.4$, respectively.

A summary of correlation coefficients of each variable is provided in Table 2. ISI score was significantly correlated with PHQ-9, FoP, and C-DBS scores, and C-DBS score was significantly correlated with patient age, sex, ISI, PHQ-9, and FoP scores. ISI and C-DBS scores were not significantly correlated with STAI-S scores.

\section{Structural Equation Model}

Fig. 1 shows our hypothesized model and Fig. 2 shows our final model after correction using multiple fitting procedures. All path coefficients in the diagram are significant. In our final model, C-DBS did not mediate PHQ's effect on ISI whereas the direct effect of PHQ on ISI was significant $(\beta=0.32, \mathrm{p}<0.001)$. C-DBS mediated the effect of FoP $(\beta=0.36, \mathrm{p}<0.001)$ and patient $\operatorname{sex}(\beta=0.13, \mathrm{p}=0.009)$ on ISI. STAI-S $(\beta=-0.09, \mathrm{p}=0.071)$ only had a direct effect on ISI (Fig. 2A). Q1-immune item and Q2-recurrence item each mediated the effect of FoP on ISI ( $\beta=$ $0.19, \mathrm{p}<0.001 ; \beta=0.23, \mathrm{p}<0.001$ ), and with Q2-recurrence item also mediating the effect of patient sex on ISI $(\beta=0.09, \mathrm{p}=0.019)$. We also discovered that Q1-immune item had a positive association with Q2-recurrence item (Fig. 2B). Patient age was included in our initial model, but was excluded in the final model because it did not show a significant correlation with either C-DBS or ISI.

\section{DISCUSSION}

C-DBS had a significant correlation with both FoP $(r=0.355)$ and ISI $(r=0.441)$, and our path analysis results suggest that it may mediate the positive effect of FoP on ISI. The Q1-immune item and the Q2-recurrence item also mediated the effect of FoP on ISI, respectively $(\beta=0.19, \mathrm{p}<0.001 ; \beta=0.23, \mathrm{p}<0.001)$. Our finding raises the possibility that C-DBS might have a critical role with regard to insomnia in cancer patients. This hints that alleviating dysfunctional beliefs as well as fear of progression could benefit cancer patients with insomnia. Given that information, intervention for dysfunctional beliefs might be an effective treatment option for cancer patients. This is in line with the results of a previous review article which investigated 12 studies that measured the effects of Cognitive-Behavior Therapy for insomnia (CBT-I) in cancer patients or survivors and CBT-I was found to be associated with significant improvement of insomnia symptoms [15]. In this regard, there is a definite need for developing CBT-I modalities specifically for cancer patients, as we have suggested earlier [16]. Our finding also advocates a cognitive behavioral approach for cancer patients with insomnia. Moreover, it suggests that patients with fear of their cancer progression may share a vulnerability for developing dysfunctional beliefs about sleep which eventually leads to insomnia.

The positive association between the Q1-immune item and the Q2-recurrence item might imply that cancer patients with insomnia have a particular thought process such as, "if I don't get good enough sleep my immune system will have problems, and this may lead to recurrence of cancer." Insomnia being affected by dysfunctional beliefs about sleep in cancer patients has been reported in several published studies $[17,18]$. Our finding raises the possibility that dysfunctional beliefs could be correlated with each other and be merged to form a consecutive thought process.

We failed to find a significant mediating effect of C-DBS between PHQ-9 and ISI, but only direct effects of PHQ-9 on ISI. The link between depression and insomnia is well known, although the underlying pathway is not fully understood. The majority of the published studies have focused on the contributing effect of insomnia in the development of depression, whereas some published studies showed that depression can be a risk factor for insomnia $[19,20]$. Insomnia has traditionally been thought to be a symptom of depression, but recent studies main-

Table 2. Correlation coefficients of each variables

\begin{tabular}{|c|c|c|c|c|c|c|c|c|}
\hline Variables & Age & Q1-immune & Q2-recurrence & C-DBS & ISI & PHQ-9 & FoP & STAI-S \\
\hline Age & 1.00 & & & & & & & \\
\hline Q1-immune & -0.046 & 1.00 & & & & & & \\
\hline Q2-recurrence & $-0.129^{*}$ & $0.689^{\dagger}$ & 1.00 & & & & & \\
\hline C-DBS & $-0.108^{*}$ & $0.913^{\dagger}$ & $0.923^{\dagger}$ & 1.00 & & & & \\
\hline ISI & 0.008 & $0.403^{\dagger}$ & $0.416^{\dagger}$ & $0.441^{\dagger}$ & 1.00 & & & \\
\hline PHQ-9 & 0.052 & $0.185^{\dagger}$ & $0.195^{\dagger}$ & $0.203^{\dagger}$ & $0.376^{\dagger}$ & 1.00 & & \\
\hline FoP & $-0.174^{\dagger}$ & $0.287^{\dagger}$ & $0.352^{\dagger}$ & $0.355^{\dagger}$ & $0.202^{\dagger}$ & $0.599^{\dagger}$ & 1.00 & \\
\hline STAI-S & $0.136^{*}$ & 0.074 & 0.078 & 0.081 & 0.024 & $0.255^{\dagger}$ & $0.269^{\dagger}$ & 1.00 \\
\hline
\end{tabular}

${ }^{*} \mathrm{p}<0.05 .{ }^{\dagger} \mathrm{p}<0.01$

C-DBS: Cancer-Related Dysfunctional Beliefs about Sleep, ISI: Insomnia Severity Index, PHQ-9: Patient Health Questionnaire-9, FoP: Fear of Progression, STAI-S: State-Trait Anxiety Inventory Score. 
tain that the two are separable and that insomnia should be regarded as a comorbid disorder [21,22].

A previous study showed that females are associated with a higher risk for developing insomnia in the general population [23], and a prospective study about sleep disruptions in patients with cancer revealed that breast cancer patients reported the highest number of overall insomnia symptoms [24]. The seemingly mediating effect of C-DBS between sex and ISI displayed in our study might imply that female patients have a tendency to develop insomnia via dysfunctional beliefs compared to male patients. Although not shown in the results, the mean Q2-recurrence item and total C-DBS scores in the female group were significantly higher than those of the male group when a t-test was performed. Taking this into consideration, a higher prevalence of dysfunctional beliefs about sleep in female cancer patients could contribute to a higher prevalence of insomnia compared to that in male cancer patients.

A few studies have explored the relationship between fear of recurrence and insomnia. One pilot study reported that intrusive thoughts about breast cancer (measured by the Impacts of Events Scale) contributed significantly to insomnia symptom severity in African-American breast cancer survivors, and accounting for $12 \%$ of the variance in ISI scores [25]. However, fear of recurrence was not a significant predictor of insomnia symp-
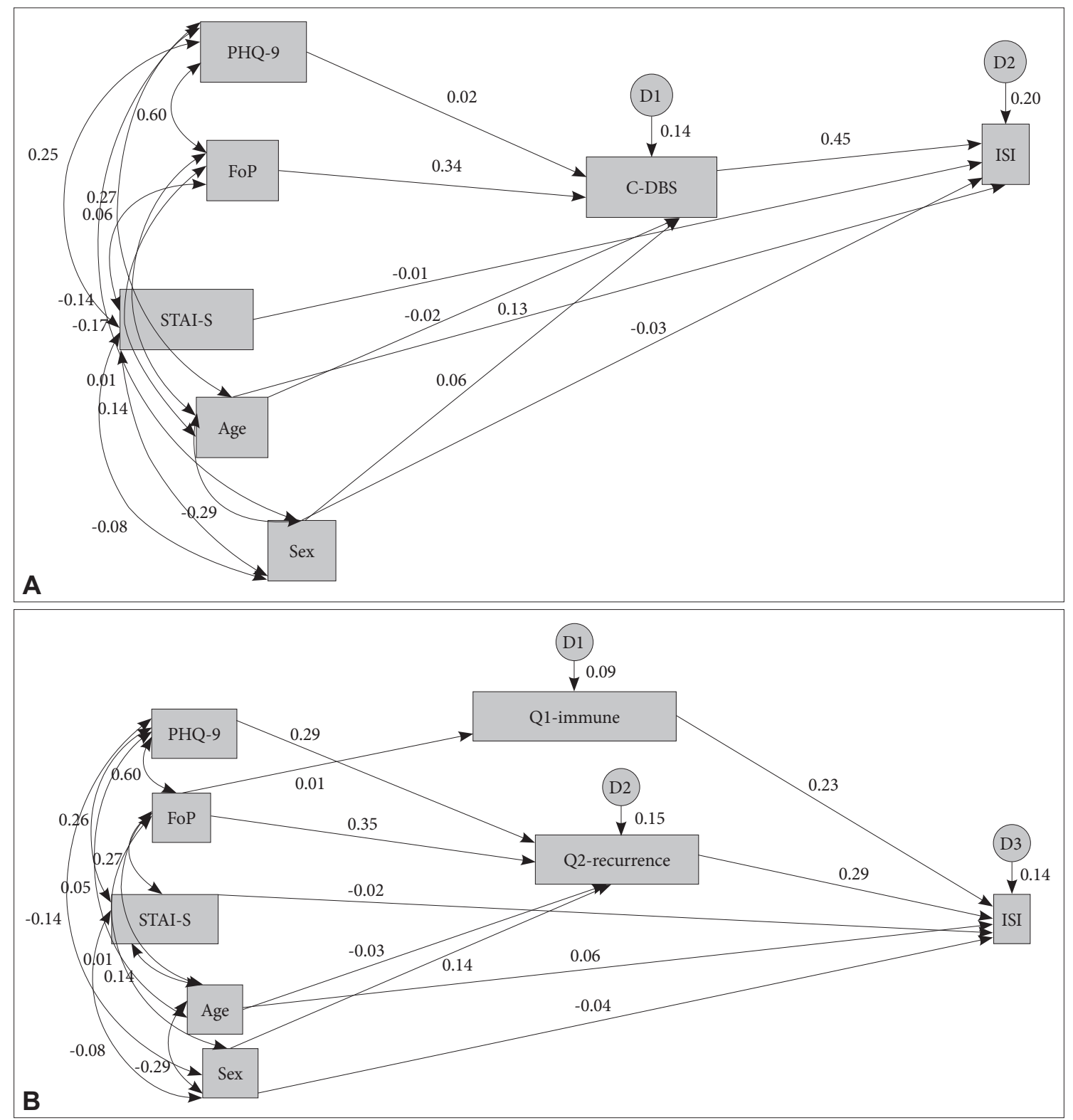

Fig. 1. Hypothetical path diagram assuming C-DBS as a possible mediator. Since C-DBS consists of two separate items, two diagrams are shown. The first one (A) with the total C-DBS score, the second one (B) with C-DBS divided into two separate item scores. C-DBS: CancerRelated Dysfunctional Beliefs about Sleep, ISI: Insomnia Severity Index, PHQ-9: Patient Health Questionnaire-9, FoP: Fear of Progression, STAI-S: State-Trait Anxiety Inventory Score. 

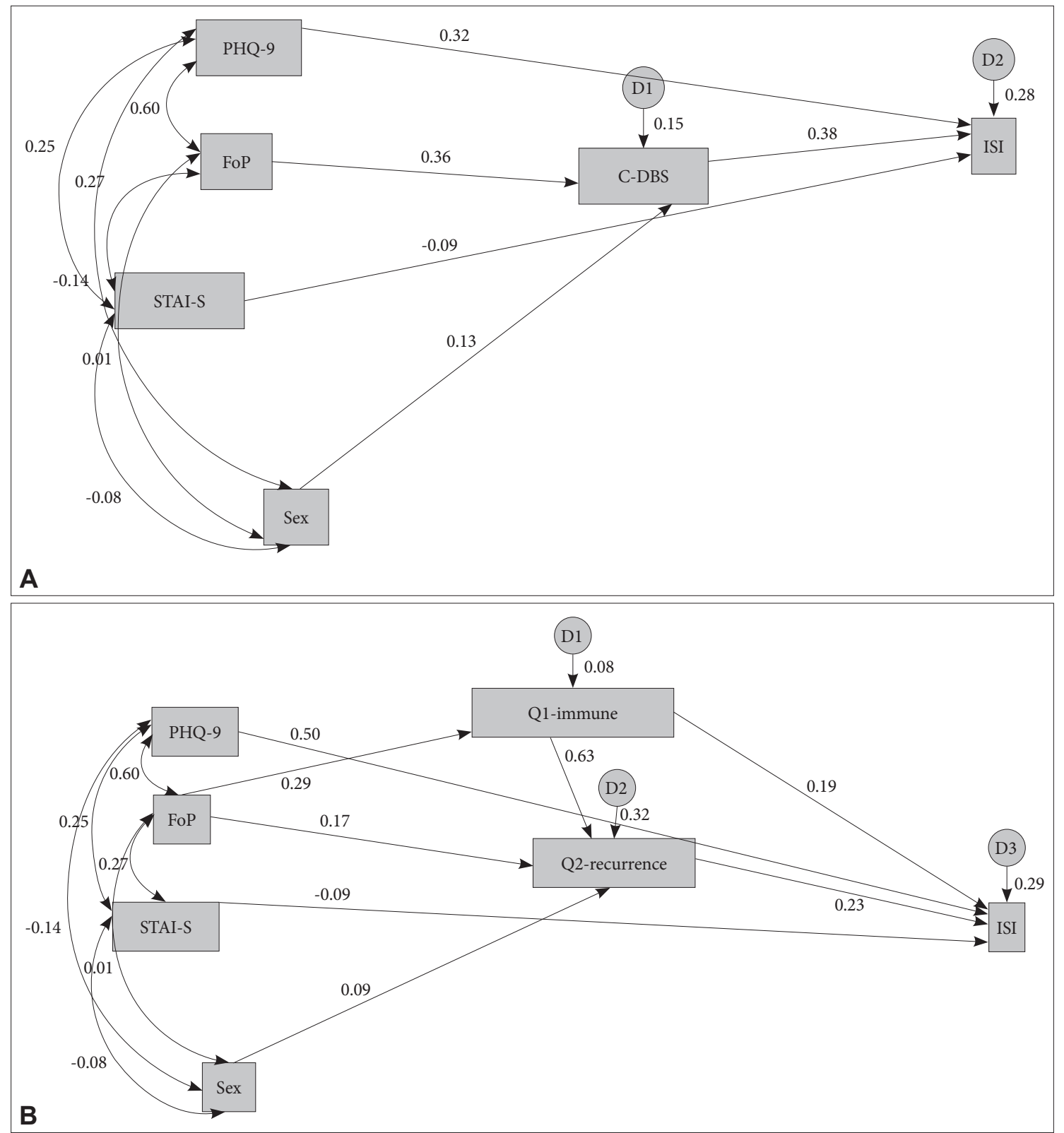

Fig. 2. Modified path diagram assuming C-DBS as a possible mediator. Since C-DBS consists of two separate items, two diagrams are shown. The first one (A) with the total C-DBS score, the second one (B) with C-DBS divided into two separate item scores. C-DBS: CancerRelated Dysfunctional Beliefs about Sleep, ISI: Insomnia Severity Index, PHQ-9: Patient Health Questionnaire-9, FoP: Fear of Progression, STAI-S: State-Trait Anxiety Inventory Score.

tom severity. In another study, higher levels of fear of recurrence had a significant relationship with poor sleep quality [8]. Fear of recurrence in the aforementioned studies was measured with the Concerns of Recurrence Scale [26], and the cancer worry subscale of the Assessment of Survivors Concerns [27], respectively. The cancer worry subscale consists of the following questions: worries about future diagnostic tests; another type of cancer; and cancer recurrence. The Concerns of Recurrence Scale has two parts, i.e., the first part measuring the overall fear of recurrence, and the second part addressing the nature of women's fears about recurrence. In our study, fear of progression was assessed using the short form of the FoP questionnaire which shares similarities with scales measuring fear of recurrence. Our study differs from the previous studies in the sense that it attempted to define the mechanism by which FoP affected insomnia severity.

There are some limitations to this study. Since it is a retrospective, cross-sectional study, casual relationships among variables cannot be inferred from our analysis. Only subjective data, i.e. questionnaire scores, were collected to evaluate psychiatric symptoms and no objective tests, such as polysomnography or actigraphy, were conducted. The variation of cancer types, stages, treat- 
ments, and phases of cancer treatment may have confounded the results and further analysis about these differences might have displayed inconsistent findings. In fact, a significant difference in the prevalence of insomnia depending on patient's cancer type was reported in a different study [24]. Also the selection bias of patients limits the generalizability of our results. It is possible that physical symptoms cancer patients experience may have influenced insomnia, but they were not assessed in our study.

Moreover, since it is a relatively newly developed scale, further studies to guarantee the validity and consistency of C-DBS should be performed.

Despite these limitations, our study was the first one to define the relationship among FoP, C-DBS, and ISI. Future research investigating how managing dysfunctional beliefs affect insomnia symptom severity in cancer patients would broaden our knowledge and benefit patients suffering from insomnia.

\section{Conflicts of Interest}

The authors have no financial conflicts of interest.

\section{Authors' Contribution}

Conceptualization: Chung S, Youn S. Data curation: Kim I, Lee J, Kim K, Choi JM. Formal analysis: Kim I, Chung S. Investigation: Chung S, Suh S. Methodology: Chung S, Yi K, Youn S, Suh S. Supervision: Chung S. Visualization: Youn S, Kim J. Writing — original draft: Kim I, Chung S. Writing—review \& editing: Yi K, Lee J, Kim K, Choi JM, Suh S, Youn S, Kim J.

\section{REFERENCES}

1. Salo P, Sivertsen B, Oksanen T, Sjösten N, Pentti J, Virtanen M, et al. Insomnia symptoms as a predictor of incident treatment for depression: prospective cohort study of 40,791 men and women. Sleep Med 2012;13:278-84.

2. Irwin MR, Olmstead RE, Ganz PA, Haque R. Sleep disturbance, inflammation and depression risk in cancer survivors. Brain Behav Immun 2013;30 Suppl:S58-67.

3. Dew MA, Hoch CC, Buysse DJ, Monk TH, Begley AE, Houck PR, et al. Healthy older adults' sleep predicts all-cause mortality at 4 to 19 years of follow-up. Psychosom Med 2003;65:63-73.

4. Chung S, Youn S, Choi B. Assessment of cancer-related dysfunctional beliefs about sleep for evaluating sleep disturbance in cancer patients. Sleep Med Res 2017;8:98-101.

5. Youn S, Kim C, Lee J, Yeo S, Suh S, Chung S. Development of dysfunctional beliefs and attitude about sleep scale for cancer patients. Behav Sleep Med 2019 Feb 21 [E-pub]. Available from: https://doi.org/10.108 0/15402002.2019.1578773.

6. Yeo S, Yi K, Kim C, Lee J, Youn S, Suh S, et al. Cancer-related dysfunctional beliefs about sleep may influence insomnia of cancer patients regardless of depressive symptoms. Sleep Med Res 2019;10:31-5.

7. Herschbach P, Dinkel A. Fear of progression. In: Goerling U. Psychooncology. Berline, Heidelberg: Springer 2014;11-29.

8. Berrett-Abebe J, Cadet T, Pirl W, Lennes I. Exploring the relationship between fear of cancer recurrence and sleep quality in cancer survivors. J Psychosoc Oncol 2015;33:297-309.

9. Bastien $\mathrm{CH}$, Vallières A, Morin CM. Validation of the Insomnia Severity Index as an outcome measure for insomnia research. Sleep Med 2001;2:297-307.

10. Cho YW, Song ML, Morin CM. Validation of a Korean version of the insomnia severity index. J Clin Neurol 2014;10:210-5.

11. Kroenke K, Spitzer RL, Williams JB. The PHQ-9: validity of a brief depression severity measure. J Gen Intern Med 2001;16:606-13.

12. Herschbach P, Berg P, Dankert A, Duran G, Engst-Hastreiter U, Waadt $\mathrm{S}$, et al. Fear of progression in chronic diseases: psychometric properties of the Fear of Progression Questionnaire. J Psychosom Res 2005;58: 505-11.

13. Shim EJ, Shin YW, Oh DY, Hahm BJ. Increased fear of progression in cancer patients with recurrence. Gen Hosp Psychiatry 2010;32:169-75.

14. Spielberger CD, Sydeman SJ. State-Trait Anxiety Inventory and StateTrait Anger Expression Inventory. In Maruish ME. The use of psychological testing for treatment planning and outcome assessment. Hillsdale NJ: Lawrence Erlbaum Associates, Inc. 1994;292-321.

15. Garland SN, Johnson JA, Savard J, Gehrman P, Perlis M, Carlson L, et al. Sleeping well with cancer: a systematic review of cognitive behavioral therapy for insomnia in cancer patients. Neuropsychiatr Dis Treat 2014;10:1113-24.

16. Youn S, Choi B, Yi K, Chung S. Cognitive-behavioral therapy for insomnia for cancer patients. Korean J Psycho-Oncol 2017;3:1-10.

17. Rumble ME, Keefe FJ, Edinger JD, Affleck G, Marcom PK, Shaw HS. Contribution of cancer symptoms, dysfunctional sleep related thoughts, and sleep inhibitory behaviors to the insomnia process in breast cancer survivors: a daily process analysis. Sleep 2010;33:1501-9.

18. Savard J, Morin CM. Insomnia in the context of cancer: a review of a neglected problem. J Clin Oncol 2001;19:895-908.

19. Katz DA, McHorney CA. Clinical correlates of insomnia in patients with chronic illness. Arch Intern Med 1998;158:1099-107.

20. Ohayon MM, Caulet M, Lemoine P. Comorbidity of mental and insomnia disorders in the general population. Compr Psychiatry 1998;39: 185-97.

21. Pigeon WR, Perlis ML. Insomnia and depression: birds of a feather? Int J Sleep Disorders 2007;1:82-91.

22. Staner L. Comorbidity of insomnia and depression. Sleep Med Rev 2010;14:35-46.

23. Ohayon MM, Roth T. What are the contributing factors for insomnia in the general population? J Psychosom Res 2001;51:745-55.

24. Palesh OG, Roscoe JA, Mustian KM, Roth T, Savard J, Ancoli-Israel S, et al. Prevalence, demographics, and psychological associations of sleep disruption in patients with cancer: University of Rochester Cancer Center-Community Clinical Oncology Program. J Clin Oncol 2010;28:292-8.

25. Taylor TR, Huntley ED, Makambi K, Sween J, Adams-Campbell LL, Frederick W, et al. Understanding sleep disturbances in AfricanAmerican breast cancer survivors: a pilot study. Psychooncology 2012; 21:896-902.

26. Vickberg SM. The Concerns About Recurrence Scale (CARS): a systematic measure of women's fears about the possibility of breast cancer recurrence. Ann Behav Med 2003;25:16-24.

27. Gotay CC, Pagano IS. Assessment of Survivor Concerns (ASC): a newly proposed brief questionnaire. Health Qual Life Outcomes 2007; 5:15. 